\title{
Weak reverse Hölder inequality of weakly A-harmonic sensors and Hölder continuity of A-harmonic sensors
}

Tingting Wang and Gejun Bao*

\footnotetext{
* Correspondence: baogj@hit.edu. cn

Department of Mathematics, Harbin Institute of Technology, Harbin 150001, PR China
}

\section{Abstract}

In this paper, we obtain the weak reverse Hölder inequality of weakly A-harmonic sensors and establish the Hölder continuity of A-harmonic sensors.

Mathematics Subject Classification 2010: 58A10 · 35J60

Keywords: Weak reverse Hölder inequality, Weakly A-harmonic sensors, Hölder continuity

\section{Introduction}

In this paper, we consider the A-harmonic equation

$$
d^{*} A(x, d u)=0,
$$

where mapping $A: \Omega \times \Lambda^{l}\left(\mathbb{R}^{n}\right) \rightarrow \Lambda^{l}\left(\mathbb{R}^{n}\right)$ satisfies the following assumptions for fixed $0<\alpha \leq \beta<\infty$ :

(1) A satisfies the Carathéodory measurability condition;

(2) for a.e. $x \in \Omega$ and all $\xi \in \Lambda^{l}\left(\mathbb{R}^{n}\right)$

$$
\langle A(x, \xi), \xi\rangle \geq \alpha|\xi|^{p}, \quad|A(x, \xi)| \leq \beta|\xi|^{p-1}
$$

(3) for a.e. $x \in \Omega$ and all $\xi \in \Lambda^{l}\left(\mathbb{R}^{n}\right), \lambda \in \mathbb{R}$

$$
A(x, \lambda \xi)=\lambda|\lambda|^{p-2} A(x, \xi)
$$

Here, $1<p<\infty$ is a fixed exponent associated with (1.1).

Remark: The notions and basic theory of exterior calculus used in this paper can be found in [1] and [2], we do not mention them here.

Definition 1.1 [2]A solution $u$ to (1.1), called A-harmonic tensor, is an element of the Sobolev space $W_{l o c}^{1, p}\left(\Omega, \Lambda^{l-1}\right)$ such that

$$
\int_{\Omega}\langle A(x, d u), d \phi\rangle \mathrm{d} x=0
$$

for all $\varphi \in W^{1, p}\left(\Omega, \Lambda^{l-1}\right)$ with compact support.

In particular, we impose the growth condition

$$
A(x, \xi) \cdot \xi \approx|\xi|^{p}
$$


then Equation (1.1) simplifies to the p-harmonic equation

$$
d^{*}\left(|d u|^{p-2} d u\right)=0
$$

The existence of the exact form $d u \in L^{p}\left(\Omega, \Lambda^{l}\right)$ is established by variational principles, and the uniqueness of $d u$ is verified by a monotonicity property of the mapping $A(x, \xi)=|\xi|^{p-2} \cdot \xi$.

We consider the following definition with exponents different from $p$.

Definition 1.2 [3]A very weak solution u to (1.1) (also called weakly A-harmonic tensor) is an element of the Sobolev space $W_{l o c}^{1, r}\left(\Omega, \Lambda^{l-1}\right)$ with $\max \{1, p-1\} \leq r<p$ such that

$$
\int_{\Omega}\langle A(x, d u), d \phi\rangle \mathrm{d} x=0
$$

for all $\phi \in W^{1, \frac{r}{r-p+1}}\left(\Omega, \Lambda^{l-1}\right)^{\text {with compact support. }}$

Compared with Definition 1.1, the Sobolev integrability exponent $r$ of $u$ in Definition 1.2 can be less than the natural Sobolev integrability exponent $p$ of the weak solution. In this case, the class of admissible test forms is considerably restricted, and it is quite difficult to derive a priori estimates. So, how to choose the test forms is especially important.

\section{Main results}

In this paper, we will present two results. The first is the weak reverse Hölder inequality for weakly A-harmonic sensors, and the second result is to establish the Hölder continuity of A-harmonic sensors.

\subsection{Weak reverse Hölder inequality of weakly A-harmonic sensors}

The reverse Hölder inequality that serves as powerful tools in mathematical analysis has many applications in the estimates of solutions. The original study of the reverse Hölder inequality can be traced back in Muckenhoupt's work in [4]. During recent years, various versions of the weak reverse Hölder inequality have been established. The weak reverse Hölder inequality for differential forms satisfying some versions of the A-harmonic equation (weighted or non-weighted) was developed by Agarwal, Ding and Nolder in [2]. In [5], there is a weak reverse Hölder inequality for very weak solutions of some classes of equations obtained by Stroffolin. And a weak reverse Hölder inequality for differential forms of the class weak $\mathcal{W} \mathcal{T}_{2}$ was proved by Gao and Wang in [3].

In this section, we establish a weak reverse Hölder inequality for weakly A-harmonic sensors. The point is to choose the appropriate test form, and the key tools in our proof are the Hodge decomposition in [6] and the Poincaré-type inequality for differential forms in [5].

Lemma 2.1 [5] Let $Q$ be a cube or a ball, and $u \in L^{r}\left(Q, \Lambda^{l}\right)$ with $d u \in L^{r}\left(Q, \Lambda^{l+1}\right)$, $1<r<\infty$. Then,

$$
\frac{1}{\operatorname{diam}(Q)}\left(\bar{\int}_{Q}\left|u-u_{Q}\right|^{r}\right)^{\frac{1}{r}} \leq c(n, r)\left(\bar{\int}_{Q}|d u|^{\frac{n r}{n+r-1}}\right)^{\frac{n+r-1}{n r}}
$$


where $\bar{\int}_{Q}$ denotes the integral mean over $Q$, that is

$$
\bar{\int}_{Q}=\frac{1}{|Q|} \int_{Q}
$$

where $|Q|$ denotes the Lebesgue measure of $Q$.

Lemma 2.2 [6] For $\omega \in L^{r(1+\varepsilon)}\left(\Omega, \Lambda^{l}\right), r \geq \frac{7}{4}$ and $\varepsilon>-\frac{1}{2}$, consider the Hodge decomposition

$$
|\omega|^{\varepsilon} \omega=d \alpha+d^{*} \beta \quad \text { with } \quad d \alpha, d^{*} \beta \in L^{r}\left(\Omega, \wedge^{l}\right) .
$$

If $\omega$ is closed (i.e. $d \omega=0$ ), then

$$
\left\|d^{*} \beta\right\|_{r} \leq c(n) r|\varepsilon|\|\omega\|_{r(1+\varepsilon)}^{1+\varepsilon} .
$$

If $\omega$ is closed (i.e. $d^{*} \omega=0$ ), then

$$
\|d \alpha\|_{r} \leq c(n) r|\varepsilon|\|\omega\|_{r(1+\varepsilon)}^{1+\varepsilon} .
$$

Our main result is the following theorem.

Theorem 2.3 Suppose that $u \in W_{l o c}^{1, r}\left(\Omega, \Lambda^{l-1}\right)$ is a weakly A-harmonic tensor, then there exists $\varepsilon_{0}>0$ such that for $|p-r|<\varepsilon_{0}$ and any cubes $Q \subset 2 Q \subset \Omega$ we have

$$
\bar{\int}_{Q}|d u|^{r} \mathrm{~d} x \leq \theta \bar{\int}_{2 Q}|d u|^{r} \mathrm{~d} x+c\left(\bar{\int}_{2 Q}|d u|^{\frac{n r}{n+r-1}}\right)^{\frac{n+r-1}{n}}
$$

where $0 \leq \theta<1, c=c(n, p, \alpha, \beta)<\infty$.

Proof: Let $\eta(x) \in C_{0}^{\infty}(2 Q)$ be a cutoff function such that $0 \leq \eta \leq 1, \eta \equiv 1$ on $Q$, and $|\nabla \eta| \leq c(n) / \operatorname{diam} Q$. Put

$$
v=\eta\left(u-u_{2 Q}\right)
$$

then there exists $\varepsilon_{1}>0$ such that for $|p-r|<\varepsilon_{1}$ the conditions of the Hodge decomposition are satisfied. So, from Lemma 2.2, we get

$$
|d v|^{r-p} d v=d \phi+h
$$

where $\phi \in W^{1, \frac{r}{r-p+1}}\left(\Omega, \Lambda^{l-1}\right), h \in L^{\frac{r}{r-p+1}}\left(\Omega, \Lambda^{l}\right)$, and

$$
\|h\|_{\frac{r}{r-p+1}} \leq c(n)|p-r|\|d v\|_{r}^{r-p+1}
$$

Write

$$
\begin{aligned}
& X=d v=d \eta \wedge\left(u-u_{2 Q}\right)+\eta d u \\
& Y=\eta d u \\
& E=|X|^{r-p} X-|Y|^{r-p} Y
\end{aligned}
$$

then by an elementary inequality in [7]

$$
\left.|| X\right|^{-\varepsilon} X-|Y|^{-\varepsilon} Y\left|\leq 2^{\varepsilon} \frac{1+\varepsilon}{1-\varepsilon}\right| X-\left.Y\right|^{1-\varepsilon}, \quad 0 \leq \varepsilon<1
$$


which also holds for differential forms, and by choosing $\varepsilon=p-r$ in (2.4), we have that

$$
|E| \leq 2^{p-r} \frac{1+p-r}{1-p+r}\left|d \eta \wedge\left(u-u_{2 Q}\right)\right|^{1-p+r}
$$

We can use $d \varphi=|d v|^{r-p} d v-h$ as a test form for the equation (1.1) to get

$$
\int_{\Omega}\left\langle A(x, d u),|d v|^{r-p} d v-h\right\rangle \mathrm{d} x=0
$$

then we obtain

$$
\int_{\Omega}\left\langle A(x, d u),|d v|^{r-p} d v\right\rangle \mathrm{d} x=\int_{\Omega}\langle A(x, d u), h\rangle \mathrm{d} x
$$

Therefore,

$$
\begin{aligned}
\int_{\Omega}\left\langle A(x, d u),|\eta d u|^{r-p} \eta d u\right\rangle \mathrm{d} x & =\int_{2 Q}\left\langle A(x, d u),|d v|^{r-p} d v-E\right\rangle \mathrm{d} x \\
& =-\int_{2 Q}\langle A(x, d u), E\rangle \mathrm{d} x+\int_{2 Q}\left\langle A(x, d u),|d v|^{r-p} d v\right\rangle \mathrm{d} x \\
& =-\int_{2 Q}\langle A(x, d u), E\rangle \mathrm{d} x+\int_{2 Q}\langle A(x, d u), h\rangle \mathrm{d} x \\
& \triangleq I_{1}+I_{2}
\end{aligned}
$$

By the (1.2), the left-hand side of this equality has the estimate

$$
\begin{aligned}
\int_{\Omega}\left\langle A(x, d u),|\eta d u|^{r-p} \eta d u\right\rangle \mathrm{d} x & =\int_{2 Q} \eta^{r-p+1}|d u|^{r-p}\langle A(x, d u), d u\rangle \mathrm{d} x \\
& \geq \int_{2 Q} \eta^{r-p+1}|d u|^{r-p} \cdot \alpha|d u|^{p} \mathrm{~d} x \\
& =\alpha \int_{2 Q} \eta^{r-p+1}|d u|^{r} \mathrm{~d} x \\
& \geq \alpha \int_{Q}|d u|^{r} \mathrm{~d} x
\end{aligned}
$$

Now we estimate $\left|I_{1}\right|$ and $\left|I_{2}\right|$. It follows from (1.2), (2.5) and Hölder inequality that

$$
\begin{aligned}
\left|I_{1}\right| & =\left|-\int_{2 Q}\langle A(x, d u), E\rangle \mathrm{d} x\right| \\
& \leq 2^{p-r} \frac{1+p-r}{1-p+r} \cdot \beta \int_{2 Q}|d u|^{p-1}|\nabla \eta|^{1-p+r}\left|u-u_{2 Q}\right|^{1-p+r} \mathrm{~d} x \\
& \leq 2^{p-r} \frac{1+p-r}{1-p+r} \cdot \beta \cdot \frac{c(n)}{(\operatorname{diam} Q)^{1-p+r}} \int_{2 Q}|d u|^{p-1}\left|u-u_{2 Q}\right|^{1-p+r} \mathrm{~d} x \\
& \leq 2^{p-r} \frac{1+p-r}{1-p+r} \cdot \beta \cdot \frac{c(n)}{(\operatorname{diam} Q)^{1-p+r}}\left(\int_{2 Q}|d u|^{r} \mathrm{~d} x\right)^{\frac{p-1}{r}} \cdot\left(\int_{2 Q}\left|u-u_{2 Q}\right|^{r} \mathrm{~d} x\right)^{\frac{r-p+1}{r}}
\end{aligned}
$$


Lemma 2.1 implies

$$
\left(\int_{2 Q}\left|u-u_{2 Q}\right|^{r} \mathrm{~d} x\right)^{\frac{1}{r}} \leq c(n, r) \cdot(\operatorname{diam} Q)^{\frac{1}{r}}\left(\int_{2 Q}|d u|^{\frac{n r}{n+r-1}} \mathrm{~d} x\right)^{\frac{n+r-1}{n r}}
$$

together with the above inequality and Young's inequality $a b \leq \varepsilon a^{p}+c(\varepsilon, p) b^{p^{\prime}}, \frac{1}{p}+\frac{1}{p^{\prime}}=1, a>0, b>0, \varepsilon>0$ we get the estimate of $\left|I_{1}\right|$ :

$$
\begin{aligned}
\left|I_{1}\right| & \leq c(n, p, r)(\operatorname{diam} Q)^{-\frac{(r-1)(r-p+1)}{r}}\left(\int_{2 Q}|d u|^{r} \mathrm{~d} x\right)^{\frac{p-1}{r}} \cdot\left(\int_{2 Q}|d u|_{\frac{n r}{n+r-1}} \mathrm{~d} x\right) \frac{(n+r-1)(r-p+1)}{n r} \\
& \leq \varepsilon \int_{2 Q}|d u|^{r} \mathrm{~d} x+c(n, p, r, \varepsilon)(\operatorname{diam} Q)^{-(r-1)}\left(\int_{2 Q}|d u|^{\frac{n r}{n+r-1}} \mathrm{~d} x\right)^{\frac{n+r-1}{n}} \\
& =\varepsilon\|d u\|_{r ; 2 Q}^{r}+c(n, p, r, \varepsilon)(\operatorname{diam} Q)^{-(r-1)} \|\left. d u\right|_{\frac{n r}{n+r-1} ; 2 Q} ^{r}
\end{aligned}
$$

Combined with (1.2), (2.3) and Hölder inequality yield

$$
\begin{aligned}
\left|I_{2}\right| & =\left|\int_{2 Q}\langle A(x, d u), h\rangle \mathrm{d} x\right| \leq \beta \int_{2 Q}|d u|^{p-1}|h| \mathrm{d} x \\
& \leq \beta\|d u\|_{r ; 2 Q}^{p-1}\|h\|_{\frac{r}{r-p+1} ; 2 Q} \\
& \leq c(n) \beta|p-r|\|d u\|_{r ; 2 Q}^{p-1}\|d v\|_{r ; 2 Q}^{r-p+1}
\end{aligned}
$$

Together with the Minkowski inequality and (2.8) yield

$$
\begin{aligned}
\|d v\|_{r ; 2 Q} & \leq\left\|d \eta \wedge\left(u-u_{2 Q}\right)\right\|_{r ; 2 Q}+\|\eta d u\|_{r ; 2 Q} \\
& \leq \frac{c(n)}{\operatorname{diam} Q}\left\|u-u_{2 Q}\right\|_{r ; 2 Q}+\|\eta d u\|_{r ; 2 Q} \\
& \leq \frac{c(n, r)}{\operatorname{diamQ}} \cdot(\operatorname{diamQ})^{\frac{1}{r}}\left(\int_{2 Q}|d u|^{\frac{n r}{n+r-1}} \mathrm{~d} x\right)^{\frac{n+r-1}{n r}}+\|d u\|_{r ; 2 Q} \\
& =c(n, r)(\operatorname{diam}(Q))^{-\frac{r-1}{r}}\|d u\|_{\frac{n r}{n+r-1} ; 2 Q}+\|d u\|_{r ; 2 Q}
\end{aligned}
$$

Thus, combined with Young's inequality we have

$$
\begin{aligned}
\left|I_{2}\right| \leq & c(n) \beta|p-r|\|d u\|_{r ; 2 Q}^{r} \\
& +c(n, p, r) \beta|p-r|(\operatorname{diam}(Q))^{-\frac{(r-1)(r-p+1)}{r}}\|d u\|_{\frac{n r}{n+r-1} ; 2 Q}^{r-p+1}\|d u\|_{r ; 2 Q}^{p-1} \\
\leq & c(n) \beta|p-r|\|d u\|_{r ; 2 Q}^{r}+\varepsilon\|d u\|_{r_{; 2} Q}^{r} \\
& +c(n, p, r, \varepsilon) \beta^{\frac{r}{r-p+1}}|p-r|^{\frac{r}{r-p+1}}(\operatorname{diam}(Q))^{-(r-1)}\|d u\|_{\frac{n r}{n+r-1} ; 2 Q}^{r}
\end{aligned}
$$


Therefore, combined (2.6)-(2.10) we get

$$
\begin{aligned}
\alpha \int_{Q}|d u|^{r} \mathrm{~d} x \leq & (2 \varepsilon+c(n) \beta|p-r|)\|d u\|_{r ; 2 Q}^{r} \\
& +\left(1+\beta^{\frac{r}{r-p+1}}|p-r|^{\frac{r}{r-p+1}}\right) c(n, p, r, \varepsilon)(\operatorname{diam}(Q))^{-(r-1)}\|d u\|_{\frac{n r}{n+r-1} ; 2 Q}^{r}
\end{aligned}
$$

Then, we have by dividing $\alpha|Q|$ in both sides that

$$
\begin{aligned}
\int_{Q}|d u|^{r} \mathrm{~d} x \leq & \frac{(2 \varepsilon+c(n) \beta|p-r|)}{\alpha} \int_{2 Q}|d u|^{r} \mathrm{~d} x \\
& +\frac{\left(1+|p-r|^{\frac{r}{r-p+1}} \beta^{\frac{r}{r-p+1}}\right) c(n, p, r, \varepsilon)}{\alpha}\left(\int_{2 Q}|d u|^{\frac{n r}{n+r-1}} \mathrm{~d} x\right)^{\frac{n+r-1}{n}}
\end{aligned}
$$

Let $\varepsilon$ small enough and we can choose $r$ close enough to $p$, i.e. there exists $0<\varepsilon_{0}<\varepsilon_{1}$ such that for sufficient small $\varepsilon$ and $|p-r|<\varepsilon_{0}$ we have $\theta=(2 \varepsilon+c(n) \beta|p-r|) / \alpha<1$, then we obtain

$$
\int_{Q}|d u|^{r} \mathrm{~d} x \leq \theta \int_{2 Q}|d u|^{r} \mathrm{~d} x+c\left(\int_{2 Q}|d u|^{\frac{n r}{n+r-1}} \mathrm{~d} x\right)^{\frac{n+r-1}{n}}
$$

where $c=c(n, p, \alpha, \beta)<\infty$. The theorem follows.

\subsection{Hölder continuity of A-harmonic sensors}

We already have the result of Hölder continuity for functions by Morrey lemma in the case of functions. In this section, we establish the Hölder continuity for differential forms satisfying A-harmonic equation (1.1) by isoperimetric inequality for differential forms from [8] and Morrey's Lemma for differential forms in [9].

Let $\Gamma=\Gamma\left(a_{1}, a_{2}\right)$ be the family of locally rectifiable arcs $\gamma \in \mathbb{R}^{n}$ joining the points $a_{1}$ and $a_{2}$. Here, $d=d\left(a_{1}, a_{2}\right)$ is the distance between the points $a_{1}, a_{2} \in \mathbb{R}^{n}$. We denote by $\mathrm{d} s$ the element of arc length in $\mathbb{R}^{n}$.

For a subdomain $D \Subset \mathbb{R}^{n}$, we set

$$
\delta(D)=\inf _{\left\{m_{k}\right\}} \liminf _{k \rightarrow \infty} d\left(m_{k}, D\right)
$$

where the infimum is taken over all possible sequences $\left\{m_{k}\right\}, m_{k} \in \mathbb{R}^{n}$, not having accumulation points in $\mathbb{R}^{n}$.

Now we give the definition of Hölder continuity for differential forms which appears in [9].

Definition 2.4 [9] Let $u$ be a differential form of degree $l$ and $D$ a compact subset of $\mathbb{R}^{n}$. We say that $u$ is Hölder continuous with exponent $\alpha$ at $a_{1} \in D$ if

$$
\inf _{\gamma \in \Gamma} \int_{\gamma}|d u| \mathrm{d} s \leq C\left(a_{1}\right) d^{\alpha}
$$


for all $a_{2} L D$ with $d=d\left(a_{1}, a_{2}\right)<(D) / 2$. One says that $u$ is Hölder continuous with exponent $\alpha$ on $D$ if (2.11) is satisfied for all $a_{1} \in D$. If $C=\sup _{a_{1} \in D} C\left(a_{1}\right)<\infty$, the differential form $u$ is called uniformly Hölder continuous on $D$.

Remark: If the differential form $u$ of degree zero, i.e. $u$ is a function, is Hölder continuous, then

$$
\left|u\left(a_{1}\right)-u\left(a_{2}\right)\right| \leq \inf _{\gamma \in \Gamma} \int_{\gamma}|\nabla u| \mathrm{d} s=\inf _{\gamma \in \Gamma} \int_{\gamma}|d u| \mathrm{d} s
$$

agrees with the usual definition for Hölder continuous functions.

Definition $2.5[10]$ A differential form $\varphi \in L_{\text {loc }}^{p}\left(\Omega, \wedge^{l}\left(\mathbb{R}^{n}\right)\right)$ is said to be weakly closed, writing $d \phi=0$, if

$$
\int_{\Omega}\left\langle\varphi, d^{*} \psi\right\rangle=0
$$

for every test form $\psi \in W_{\text {loc }}^{1, p^{\prime}}\left(\Omega, \wedge^{l+1}\left(\mathbb{R}^{n}\right)\right)$ with compact support contained in $\Omega$, where the exponent $p^{\prime}$ is the Hölder conjugate of $p$.

Remark: For smooth differential forms $\phi$, the definition above agrees with the traditional definition of closedness $d \phi=0$.

Definition 2.6 [8]A pair of weakly closed differential forms $\Phi \in L^{r}\left(\Omega, \Lambda^{l}\left(\mathbb{R}^{n}\right)\right)$ and $\Psi \in L^{s}\left(\Omega, \Lambda^{n-1}\right)$, where $1<r, s<\infty$ satisfy Sobolev's relation $\frac{1}{r}+\frac{1}{s}=1+\frac{1}{n^{\prime}}$ will be called an admissible pair if $\Phi \Lambda \Psi \geq 0$ and

$$
\liminf _{t \rightarrow \infty} t^{\frac{1}{n}} \int_{H>t} H(x) \mathrm{d} x=0
$$

where $H=|\Phi|^{r}+|\Psi|^{s}$.

Remark: Inequality between two volume forms should be understood as inequality between their coefficients with respect to the standard basis, that is to say, we say that an $n$-form $\alpha$ on $\mathbb{R}^{n}$ is nonnegative if $\alpha=\lambda d x$ for some nonnegative function $\lambda$.

The main lemmas we used are the following

Lemma 2.7 [8]Let $(\Phi, \Psi)$ be an admissible pair. Given $x \in \mathbb{R}^{n}$, for almost every all $B$ $=B(x, \delta) \subset \mathbb{R}^{n}, 0<\delta<\delta(D) / 2$ we have

$$
\int_{B} \Phi \wedge \Psi \leq c(n)\left(\int_{\partial B}\left(|\Phi|^{r}+|\Psi|^{s}\right)\right)^{\frac{n}{n-1}}
$$

provided $1<s=\frac{r(n-1)}{n r-n+1}$.

Lemma 2.8 [9] (Morrey's Lemma) Let $u \in W_{l o c}^{1, p}\left(\Omega, \Lambda^{l}\left(\mathbb{R}^{n}\right)\right), 0 \leq l \leq n$. If for each point $a \in D$ and $r<\delta(D) / 2$ the equality

$$
\int_{B(a, r)}|d u|^{p} \leq C r^{n-p+\alpha}
$$


holds, then for all $a_{1}, a_{2} \in D, d\left(a_{1}, a_{2}\right)<\delta(D) / 2$, we get

$$
\inf _{\gamma \in \Gamma\left(a_{1}, a_{2}\right)} \int_{\gamma}|d u| \mathrm{d} s \leq C d^{\alpha / p},
$$

where $C=C(n, p, \alpha)$.

As an application of the isoperimetric inequality (2.12) and the Morrey's Lemma 2.8, we establish the Hölder continuity of A-harmonic sensors. Namely, we have the following

Theorem 2.9 Suppose that a differential form $u \in W_{l o c}^{1, p}\left(\Omega, \Lambda^{l}\left(\mathbb{R}^{n}\right)\right)$ with $\frac{n}{n-1}<p<n$, is A-harmonic, then $u$ is Hölder continuous.

Proof: Firstly, we set $\Phi=d u, \Psi=* A(x, d u)$. We should to prove $(\Phi, \Psi)$ is an admissible pair. It is easy to see that $\Phi$ is closed, so it is weakly closed. And the weak closedness of $* A(x, d u)$ follows from

$$
\begin{aligned}
(-1)^{n l+1} \int_{\Omega}\left\langle * A(x, d u), d^{*} \psi\right\rangle & =\int_{\Omega}\langle * A(x, d u), * d * \psi\rangle \\
& =\int_{\Omega}\langle A(x, d u), d * \psi\rangle=\int_{\Omega}\langle A(x, d u), * d \phi\rangle=0
\end{aligned}
$$

for all $\psi=(-1)^{l(n-l)} * \phi \in W_{0}^{1, q}\left(\Omega, \Lambda^{n-l+1}\left(\mathbb{R}^{n}\right)\right)$. Next we set $r=\frac{p n}{n+1}$ and $s=\frac{p^{\prime} n}{n+1}$ in Definition 2.6, where the exponent $p^{\prime}$ is the Hölder conjugate of $p$. Then, we have $\frac{1}{r}+\frac{1}{s}=1+\frac{1}{n}$ and

$$
\begin{aligned}
H=|\Phi|^{r}+|\Psi|^{s} & =|d u|^{\frac{p n}{n+1}}+|A(x, d u)|^{\frac{p^{\prime} n}{n+1}} \\
& \leq|d u|^{\frac{p n}{n+1}}+\beta^{\frac{p^{\prime} n}{n+1}}|d u|^{\frac{p n}{n+1}} \\
& =c(n, p, \beta)|d u|^{\frac{p n}{n+1}} \in L^{\frac{n+1}{n}}
\end{aligned}
$$

thus we have

$$
\begin{aligned}
0 \leq t^{\frac{1}{n}} \int_{H>t} H(x) \mathrm{d} x & =\int_{H>t} t^{\frac{1}{n}} H(x) \mathrm{d} x \\
& \leq \int_{H>t} H(x)^{\frac{n+1}{n}} \mathrm{~d} x
\end{aligned}
$$

tends to 0 as $t$ tends to $\infty$. Moreover, since

$$
\Phi \wedge \Psi=d u \wedge * A(x, d u)=\langle A(x, d u), d u\rangle * 1 \geq \alpha|d u|^{p} * 1
$$

we get by Definition 2.6 that $(\Phi, \Psi)$ is an admissible pair.

Secondly, we set $1<r=\frac{p(n-1)}{n}<n-1$ and $s=\frac{p^{\prime}(n-1)}{n}$ in (2.12), then we have $s=\frac{r(n-1)}{n r-n+1}>1$ and by applying the isoperimetric inequality (2.12) for the admissible pair $(\Phi, \Psi)$, we obtain that 


$$
\begin{aligned}
& \alpha \int_{B(a, r)}|d u|^{p} \leq \int_{B(a, r)} d u \wedge * A(x, d u) \\
& \leq c(n)\left(\int_{\partial B(a, r)}\left(|d u|^{r}+|* A(x, d u)|^{s}\right)\right)^{\frac{n}{n-1}} \\
& =c(n)\left(\int_{\partial B(a, r)}\left(|d u|^{r}+|A(x, d u)|^{s}\right)\right)^{\frac{n}{n-1}} \\
& \leq c(n)\left(\int_{\partial B(a, r)}\left(|d u|^{r}+\beta^{s}|d u|^{s(p-1)}\right)\right)^{\frac{n}{n-1}} \\
& =c(n)\left(\int_{\partial B(a, r)}\left(|d u|^{r}+\beta^{s}|d u|^{r}\right)\right)^{\frac{n}{n-1}} \\
& =c(n)\left(1+\beta^{s}\right)^{\frac{n}{n-1}}\left(\int_{\partial B(a, r)}|d u|^{\frac{p(n-1)}{n}}\right)^{\frac{n}{n-1}} \\
& \leq c(n)\left(1+\beta^{s}\right)^{\frac{n}{n-1}}\left(\int_{\partial B(a, r)}|d u|^{p}\right) \cdot\left(\int_{\partial B(a, r)}\right)^{\frac{1}{n-1}} \\
& =c(n)\left(1+\beta^{s}\right)^{\frac{n}{n-1}}\left(n \omega_{n}\right)^{\frac{1}{n-1}} r \int_{\partial B(a, r)}|d u|^{p}
\end{aligned}
$$

Therefore,

$$
\int_{0}^{r} \mathrm{~d} t \int_{\partial B(a, t)}|d u|^{p}=\int_{B(a, r)}|d u|^{p} \leq \frac{c(n)\left(1+\beta^{s}\right)^{\frac{n}{n-1}}\left(n \omega_{n}\right)^{\frac{1}{n-1}}}{\alpha} r \int_{\partial B(a, r)}|d u|^{p}
$$

Setting

$$
h(r)=\int_{0}^{r} \mathrm{~d} t \int_{\partial B(a, t)}|d u|^{p}
$$

then

$$
h(r) \leq C_{0} r h(r)^{\prime},
$$

where $C_{0}=c(n)\left(1+\beta^{s}\right)^{\frac{n}{n-1}}\left(n \omega_{n}\right)^{\frac{1}{n-1}} / \alpha$. Then, we have $\left(r^{-\frac{1}{C_{0}}} h(r)\right)^{\prime} \geq 0$, therefore $r^{-\frac{1}{C_{0}}} h(r)$ is increasing, then we get $h(r) \leq\left(\frac{r}{\delta}\right)^{\frac{1}{C_{0}}} h(\delta)$, i.e.

$$
\int_{B(a, r)}|d u|^{p} \leq\left(\frac{r}{\delta}\right)^{\frac{1}{C_{0}}} \int_{B(a, \delta)}|d u|^{p}=C r^{\frac{1}{C_{0}}}
$$


Therefore, the Morrey's lemma (Lemma 2.8) infers that

$$
\inf _{\gamma \in \Gamma\left(a_{1}, a_{2}\right)} \int_{\gamma}|d u| \mathrm{d} s \leq C d^{1-\frac{n}{p}+\frac{1}{C_{0} p}}
$$

i.e. $u$ is Hölder continuous with the exponent $1-\frac{n}{p}+\frac{1}{C_{0} p}$. The theorem follows.

\section{Acknowledgements}

This work was supported by the National Natural Science Foundation of China (Grant No. 11071048).

\section{Authors' contributions}

All authors contributed equally in this paper. They read and approved the final manuscript.

\section{Competing interests}

The authors declare that they have no competing interests.

Received: 13 April 2011 Accepted: 27 October 2011 Published: 27 October 2011

\section{References}

1. Iwaniec, T, Lutoborski, A: Integral estimates for null Lagrangians. Arch Ration Mech Anal. 125, 25-79 (1993). doi:10.1007/ BF00411477

2. Agarwal, RP, Ding, S, Nolder, C: Inequalities for Differential Forms. Springer, New York (2009)

3. Gao, H, Wang, Y: Weak $\mathcal{W} \mathcal{T}_{2}$-class of differential forms and weakly A-harmonic tensors. Appl Math J Chin Univ. 25(30), 359-366 (2010). doi:10.1007/s11766-010-2292-z

4. Muckenhoupt, B: Weighted norm inequalities for the Hardy maximal function. Trans Am Math Soc. 165, $207-226$ (1972)

5. Stroffolin, B: On weakly A-harmonic tensors. Studia Math. 114(30), 289-301 (1995)

6. Iwaniec, T: p-Harmonic tensors and quasiregular mappings. Ann Math. 136, 589-624 (1992). doi:10.2307/2946602

7. Iwaniec, T, Migliaccio, L, Nania, L, Sbordone, C: Integrability and removability results for quasiregular mappings in high dimensions. Math Scand. 75, 263-279 (1994)

8. Giannetti, F, Passarelli di Napoli, A: Isoperimetric type inequalities for differential forms on mainfolds. J Indiana Univ Math. 54(50), 1483-1498 (2005). doi:10.1512/iumj.2005.54.2665

9. Franke, DC: Quasiregular Mappings and Hölder Continuity of Differential Forms on Riemannian Manifolds. Freie Universität, Berlin (1999)

10. Franke, D, Martio, O, Miklyukov, VM, Vuorinen, $M$, Wisk, R: Quasiregular mappings and $\mathcal{W} \mathcal{T}$-classes of differential forms on Riemannian mainfolds. Pac J Math. 1(2020), 73-92 (2002)

doi:10.1186/1029-242X-2011-99

Cite this article as: Wang and Bao: Weak reverse Hölder inequality of weakly A-harmonic sensors and Hölder continuity of A-harmonic sensors. Journal of Inequalities and Applications 2011 2011:99.

\section{Submit your manuscript to a SpringerOpen ${ }^{\circ}$ journal and benefit from:}

- Convenient online submission

Rigorous peer review

- Immediate publication on acceptance

- Open access: articles freely available online

- High visibility within the field

- Retaining the copyright to your article

Submit your next manuscript at $>$ springeropen.com 